\title{
A Fresh Look at the Level of Unmet Need for Family Planning in the Postpartum Period, Its Causes And Program Implications
}

One central rationale for the promotion of family planning is the potential benefit to the health of mothers and children. The achievement of adequate spacing between pregnancies is a major pathway through which contraceptive practice can render benefits. Pregnancies conceived less than 20 months following a prior birth are at considerably higher risk of prematurity, low birth weight, fetal death and early neonatal death. ${ }^{1}$ The importance of this link is underscored by the facts that neonatal deaths account for $44 \%$ of all childhood deaths and that prematurity is the most common cause. ${ }^{2}$ In addition to the 2.9 million neonatal deaths that occur each year, 99\% in low- and middleincome countries, an almost equal number of stillbirths occur. $^{3}$ An analysis of Demographic and Health Survey (DHS) data from 52 countries showed that the adverse effects of inadequate spacing extend beyond the neonatal period. ${ }^{4}$ Expressed in terms of interbirth intervals, children born within 24 months of an elder sibling have a $60 \%$ increased risk of dying before their first birthday and those born 2-3 years after an elder sibling have a 10\% increased risk, compared with those born after an interval of 3-5 years. For children between the ages of one and five years, the balance of evidence suggests that intervals of less than two years are associated with a $40 \%$ increased risk of dying. A systematic review confirmed that the health of mothers is also affected by inadequate spacing. ${ }^{5}$ Specifically, short intervals are associated with uterine rupture and uteroplacental bleeding disorders. Despite the steep rise in contraceptive use in developing countries, interbirth intervals have increased in length only slightly over the past 25 years; according to recent surveys, $25 \%$ of second and higher-order children are born within two years of a sibling, compared with about $29 \%$ a decade earlier. ${ }^{6}$

Though this evidence underlines the importance of postpartum family planning for the health of both mothers and children, the topic suffered a prolonged era of benign neglect that has only recently been remedied. In 2013, the World Health Organization issued new guidelines for this component of contraceptive services and the U.S. Agency for International Development is funding projects to provide postpartum services. ${ }^{7,8}$ One factor that helped to revitalize this agenda was a study of women in 27 countries by Ross and Winfrey in which the proportion who wanted to postpone another child for at least two years but were using no method of contraception was, on average, at the very high level of $65 \%$ in the first year following childbirth. ${ }^{9}$ Indeed, their analysis suggested that $39 \%$ of all unmet need for contraception arose from women who had given birth in the previous 12 months.

For decades, the concept of unmet need has been the main way of prioritizing populations for delivery of family planning services, and thus Ross and Winfrey's article had a major impact. However, their findings are perplexing because it is well established that most couples wish to space successive children by at least two years ${ }^{6}$ and that a woman's contact with health services is especially frequent during pregnancy, delivery and in the first year of a child's life. Thus information about, demand for and access to contraceptive services should be high in this phase of the reproductive cycle. This conundrum provided the motivation for this study. Our aims are to reassess the level of postpartum unmet need with different methods of measurement, compare unmet need in the first year following childbirth with unmet need at later durations postpartum and to analyze reasons for postpartum unmet need.

\section{Estimating Unmet Need for Contraception Among Postpartum Women}

Defining unmet need in postpartum women encounters the problem that many are wholly or partially protected by lactational amenorrhea or postnatal abstinence and thus have a reason to postpone contraception regardless of their future childbearing wishes. The standard DHS solution is to classify amenorrheic women according to the intendedness of their most recent birth. ${ }^{10}$ Postnatal abstinence, an important factor in some West African countries, is ignored. For aggregate estimates, this approach of classifying amenorrheic women is preferable to their omission from estimates, but it is unsatisfactory for describing need at the time of the survey. It also has the disadvantage that unmet need in postpartum women cannot be easily compared with that of other women, because women in the former group are much more likely to be classified on their retrospective preferences than are those in the latter group, for whom unmet need is based largely on their future childbearing preferences.

An alternative was introduced by Ross and Winfrey, who argued that amenorrheic women may not have a pressing current need for contraception, but many will soon have such a need and thus can be usefully classified as having unmet need unless they are using a contraceptive method. ${ }^{9}$ Under this "prospective" approach, women who are amenorrheic or still abstaining since their last birth are classified as having unmet need unless they want a child within two years or are using a traditional or modern method.

\section{By John Cleland, lqbal H. Shah and Lenka Benova}

\section{John Cleland is} emeritus professor of medical demography and Lenka Benova is lecturer, both at the Department of Population Health, Faculty of Epidemiology and Population Health, London School of Hygiene \& Tropical Medicine. Iqbal H. Shah is director of the Center for Research and Evaluation in Reproductive Health, Harvard School of Public Health, Boston, MA, USA. 
TABLE 1. Percentage of married or cohabiting women at 0-11 months postpartum with unmet need for any method of contraception, by measure; and selected background indicators, by region and country

\begin{tabular}{|c|c|c|c|c|c|c|c|}
\hline \multirow[t]{2}{*}{ Country (Abbreviation) } & \multirow{2}{*}{$\begin{array}{l}\text { Survey } \\
\text { Year }\end{array}$} & \multicolumn{3}{|c|}{ Measure of unmet need } & \multicolumn{3}{|c|}{ Selected background indicators $†$} \\
\hline & & DHS & $\begin{array}{l}\text { Current } \\
\text { Status }\end{array}$ & Prospective & $\begin{array}{l}\text { Total } \\
\text { fertility } \\
\text { rate }\end{array}$ & $\begin{array}{l}\% \text { birth } \\
\text { intervals } \\
<24 \text { mos. }\end{array}$ & $\begin{array}{l}\text { Median mos. } \\
\text { of postpartum } \\
\text { insusceptibility }\end{array}$ \\
\hline \multicolumn{8}{|l|}{ West/Central Africa } \\
\hline Dem.Rep.of Congo (CD) & 2007 & 32.4 & 8.3 & 61.2 & 6.3 & 26.0 & 12.1 \\
\hline Nigeria (NG) & 2008 & 19.5 & 8.9 & 65.3 & 5.7 & 23.8 & 13.8 \\
\hline Republic of Congo (CG) & 2005 & 28.6 & 3.9 & 53.7 & 4.8 & 13.2 & 11.8 \\
\hline Ghana (GH) & 2008 & 50.6 & 10.9 & 82.0 & 4.0 & 14.0 & 12.4 \\
\hline \multicolumn{8}{|l|}{ East/Southern Africa } \\
\hline Uganda (UG) & 2011 & 47.5 & 13.2 & 75.1 & 6.2 & 25.3 & 11.0 \\
\hline Kenya (KE) & $2008-9$ & 36.7 & 11.3 & 63.2 & 4.6 & 22.6 & 10.3 \\
\hline Zimbabwe (ZW) & 2010-11 & 14.6 & 3.0 & 26.1 & 4.1 & 8.8 & 12.7 \\
\hline Lesotho (LS) & 2009 & 40.4 & 8.6 & 57.8 & 3.3 & 12.4 & 11.5 \\
\hline \multicolumn{8}{|l|}{ North Africa/Asia } \\
\hline Pakistan (PK) & $2012-13^{*}$ & 30.5 & 19.3 & 52.9 & 3.8 & 36.6 & 4.4 \\
\hline Jordan (JO) & $2012^{*}$ & 14.8 & 10.5 & 25.4 & 3.5 & 31.9 & 3.1 \\
\hline Indonesia (ID) & 2012 & 12.6 & 6.2 & 27.9 & 2.6 & 10.5 & 3.8 \\
\hline Morocco (MO) & $2003-4^{*}$ & 13.1 & 6.2 & 23.2 & 2.5 & 16.6 & 3.1 \\
\hline \multicolumn{8}{|l|}{ Latin America/Caribbean } \\
\hline Guyana (GY) & 2009 & 39.8 & 21.3 & 57.4 & 2.8 & 24.8 & 4.3 \\
\hline Dominican Rep (DR) & 2007 & 20.8 & 12.3 & 27.7 & 2.4 & 23.4 & 3.4 \\
\hline Peru (PE) & 2012 & 18.9 & 3.0 & 31.9 & 2.6 & 11.8 & 10.4 \\
\hline Honduras (HN) & $2011-12$ & 13.5 & 3.7 & 27.2 & 2.9 & 15.3 & 8.6 \\
\hline
\end{tabular}

A third strategy for estimating unmet need in the postpartum period is to restrict unmet need to women who have resumed menses and sex, are not practicing contraception, but wish to postpone childbearing for two or more years. In other words, amenorrheic or abstaining women are classified along with women practicing contraception and those who want another child within two years as having no unmet need. This "current-status" approach comes closer than the standard DHS definition to capturing women's immediate need for contraception, though it is subject to the criticism that reliance on amenorrhea is not $100 \%$ effective.

To determine how the use of these differing definitions affects the level of unmet need in the postpartum period, we analyzed data from 16 countries that had a recent DHS. The countries were selected purposively to obtain a geographic spread and equal numbers with high and low prevalence of short interbirth intervals. In Table 1 , the first two countries in each regional group have a high prevalence and the second two a low prevalence of short intervals. We then applied the three measures of unmet need and compared the results.

The three measures yield very different results for women 0-11 months postpartum (Table 1). The denominator for all three estimates is currently married (or cohabiting) women, and all three categorize the small minority (about $3 \%$ ) of currently pregnant women according to the intendedness of their pregnancy; that is, those who reported the pregnancy as mistimed or unwanted were classified as part of the group with unmet need. The prospective measure gives the highest estimates of unmet need for any meth- od, ranging from $23 \%$ in Morocco to $82 \%$ in Ghana. The mean for all 16 surveys is $47 \%$. These estimates are similar to those given by Ross and Winfrey for a different set of surveys and are the origin of the assertion that unmet need is particularly high in the postpartum period. ${ }^{9}$ The current-status method gives the lowest estimates, with a range of $3-21 \%$ and a mean of $9 \%$. In every survey, the DHS definition yields values that are intermediate. Depending on which approach is preferred, unmet need in the 12 months postpartum may be regarded as exceptionally high or exceptionally low-an unhelpful situation.

The prospective definition has influenced the priorities of major donors and helped revitalize the postpartum family planning agenda, which had been neglected since the end of the Population Council's International Postpartum Program in 1974. ${ }^{7,11,12}$ It can be argued that the promotion of highly effective long-acting reversible methods (LARC) or sterilization early in the postpartum should be a top priority because short interbirth intervals, with their wellestablished adverse health consequences, remain common. As mentioned in the introduction, an analysis of DHS data from 72 countries found that $25 \%$ of interbirth intervals, on average, were shorter than 24 months. ${ }^{6}$ This strong health rationale may justify early postpartum promotion of methods even in settings where prolonged breast-feeding and amenorrhea act to postpone pregnancy. Provided that method continuation is high, the problem of redundant protection stemming from the overlap between amenorrhea and contraceptive use can be dismissed.

Conversely, postpartum uptake within a few months of childbirth of methods associated with low continuation, 
such as oral contraceptives and injectables, may have little effect, or even be counterproductive, in terms of birthspacing in populations with prolonged lactational amenorrhea. In Bangladesh, early adopters of oral contraceptives experienced higher pregnancy risks than nonusers because of discontinuation of use around the time that they became exposed to the risk of pregnancy; ${ }^{13}$ in Peru and Indonesia, women who started use of pills and (more surprisingly) IUDs in the first six months postpartum were more likely to experience pregnancy within 24 months than those adopting at 6-11 months postpartum. ${ }^{14}$ An overlap of contraceptive use with amenorrhea, together with high discontinuation, may be responsible for the finding that, in four countries of Sub-Saharan Africa, use of injectables was associated with a shortening of birth intervals at an aggregate level. ${ }^{15}$

Assessment of the merits of the current-status definition raises a central question about postpartum family planning. Beyond the strict conditions of the lactational amenorrhea method (LAM), to what extent should the protective effect of amenorrhea be acknowledged in policies and programming? In the first six months postpartum, amenorrheic women have a very low cumulative chance of conception, even if they are not fully breast-feeding, because a large fraction of first cycles are anovulatory. ${ }^{16,17}$ Rigid adherence to LAM conditions makes little difference in these first six months, provided breast-feeding is frequent. The protective effect of amenorrhea progressively weakens as duration postpartum lengthens. Nevertheless, a review of nine studies by Kennedy and Visness concluded that, among sexually active, amenorrheic, breast-feeding women who were not practicing contraception, the cumulative 12 -month pregnancy rate was only $6 \%$ on average. ${ }^{18}$ This level of risk is not much different from that of oral contraceptives or condoms under real life conditions, though, of course, most failure with pills and condoms can be attributed to incorrect use, whereas conception during amenorrhea is a matter of biological chance. The estimate of Kennedy and Visness is also consistent with the percentage of women in the 16 countries listed in Table 1 who, in answer to a direct question, stated that they became pregnant with the most recent birth before the return of menses. These range from $2 \%$ to $11 \%$ (data not shown).

It is also clear from examination of links between amenorrhea and contraception that many women in low- and middle-income countries await the return of menses before starting a method. ${ }^{12,19,20}$ At 6-9 months postpartum, menstruating women are two to three times more likely to be using a modern method as women whose menses have not yet returned. ${ }^{21}$ To assess whether the link between the return of menses and contraceptive initiation is the result of conscious decisions, we used DHS data from our 16 selected countries to examine reasons for nonuse stated by amenorrheic women who had resumed sex. Two interrelated responses, breast-feeding and amenorrhea, were the most commonly mentioned in 14 of the 16 surveys, comprising $60 \%$ or more of all reasons in seven surveys
TABLE 2. Among amenorrheic, sexually active women at 0-23 months postpartum who did not want another child in the next two years, percentage who gave selected reasons for nonuse of contraceptives, by region and country

\begin{tabular}{|c|c|c|c|c|c|c|}
\hline Country & $\begin{array}{l}\text { Amen- } \\
\text { orrhea } \\
\%\end{array}$ & $\begin{array}{l}\text { Breast- } \\
\text { feeding } \\
\%\end{array}$ & $\begin{array}{l}\text { Health } \\
\text { concerns/ } \\
\text { side effects } \\
\%\end{array}$ & $\begin{array}{l}\text { Oppo- } \\
\text { sition } \\
\%\end{array}$ & $\begin{array}{l}\text { Lack of } \\
\text { access/ } \\
\text { knowledge } \\
\%\end{array}$ & $\begin{array}{l}\text { Infre- } \\
\text { quent } \\
\text { sex } \\
\%\end{array}$ \\
\hline \multicolumn{7}{|l|}{ West/Central Africa } \\
\hline Dem.Rep.of Congo & 14 & 27 & 19 & 26 & 23 & 8 \\
\hline Nigeria & 1 & 18 & 9 & 39 & 21 & 2 \\
\hline Rep.of Congo & 27 & 25 & 11 & 7 & 18 & 6 \\
\hline Ghana & 12 & 29 & 29 & 18 & 4 & 8 \\
\hline \multicolumn{7}{|l|}{ East/Southern Africa } \\
\hline Uganda & 38 & 29 & 24 & 16 & 8 & 4 \\
\hline Kenya & 10 & 20 & 26 & 19 & 13 & 4 \\
\hline Zimbabwe & 8 & 30 & 13 & 28 & 5 & 8 \\
\hline Lesotho & 23 & 21 & 15 & 14 & 13 & 12 \\
\hline \multicolumn{7}{|l|}{ North Africa/Asia } \\
\hline Pakistan & 53 & 26 & 10 & 13 & 4 & 6 \\
\hline Jordan & 34 & 36 & 22 & 4 & 2 & 14 \\
\hline Indonesia & 21 & 22 & 13 & 3 & 3 & 8 \\
\hline Morocco & 53 & 8 & 12 & 7 & 0 & 18 \\
\hline \multicolumn{7}{|c|}{ Latin America/Caribbean } \\
\hline Guyana & 2 & 14 & 23 & 12 & 7 & 7 \\
\hline Dom.Rep. & 25 & 39 & 15 & 10 & 4 & 6 \\
\hline Peru & 44 & 19 & 12 & 4 & 3 & 21 \\
\hline Honduras & 24 & 38 & 8 & 26 & 7 & 16 \\
\hline
\end{tabular}

and more than $40 \%$ in a further five (Table 2). These responses were less dominant in Nigeria, where opposition to contraception was frequently cited; in Kenya and Guyana, where health concerns and side effects were mentioned by many women; and in Zimbabwe, where religious prohibitions and fear of side effects were common reasons. Two Indian studies found similar results. ${ }^{22,23}$ The conclusion is clear: Women consciously delay contraception until the return of menses, or perhaps until their child is weaned.

To investigate the possibility that breast-feeding may act as a deterrent to contraceptive adoption in addition to amenorrhea, we compared modern method use among breast-feeding women and women who had weaned their child, restricting attention to those who had resumed sex and menstruation (Figure 1, page 158). Interpretation should be cautious because only small minorities of women had stopped breast-feeding. Nevertheless, the results for the eight countries in Sub-Saharan Africa are consistent. In all cases, differences are positive, indicating higher use among breast-feeding women than among other women. This behavioral evidence appears to be inconsistent with the self-reported reasons in Table 2. As reported behavior usually trumps reported reasons for behavior in terms of credibility, it is likely that women citing breast-feeding as a reason for nonuse are really referring to lack of menses induced by breast-feeding. In the other eight countries, no clear-cut pattern is apparent. The largest difference occurs in Morocco, where the method mix is dominated by oral contraceptives. In this country, breast-feeding women are much less likely to be users than other women. 
FIGURE 1. Differences in absolute percentage points in modern method use between breast-feeding and non-breast-feeding women at 0-11 months postpartum who had resumed sex and menstruation, by region and country

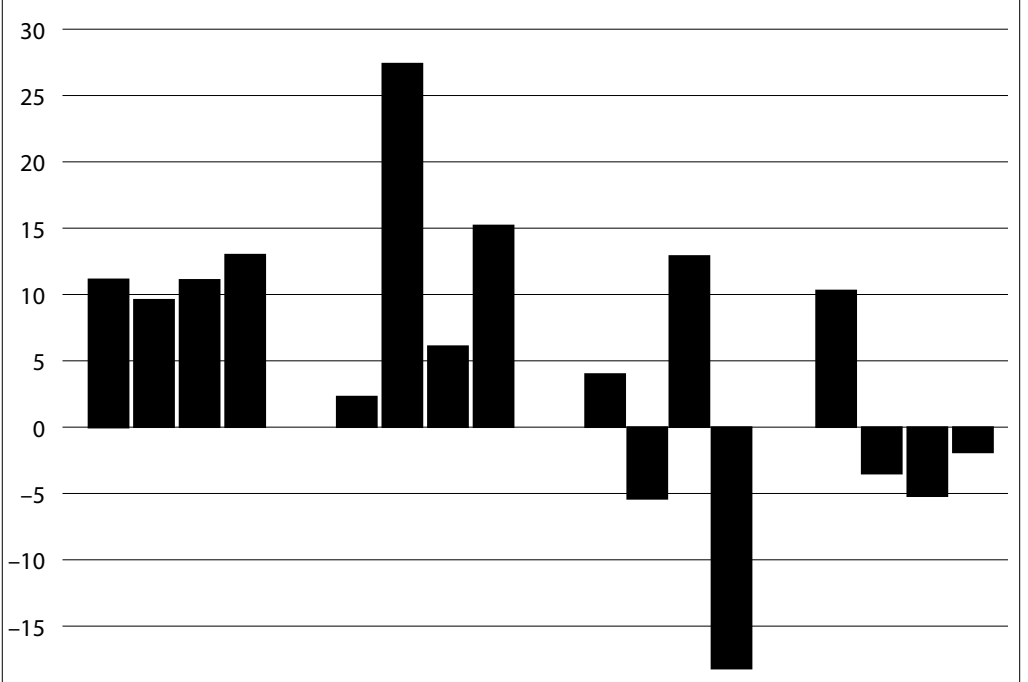

$-20$

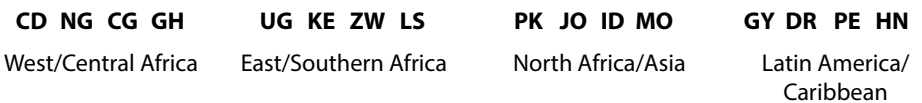

Notes: The reference group (coded 0) consists of women who are not breast-feeding. See Table 1 for country abbreviations. and infant, and they regarded amenorrhea as a means by which the woman's body recovered from the strain of pregnancy and childbirth. The onset of menses was understood to mean the return of reproductive capacity. The possibility of conception during amenorrhea was recognized, but the risk was considered small. Reluctance to start modern methods before the return of menses was reinforced by the belief that the methods were "strong" and even somewhat hazardous, particularly in the vulnerable months after childbirth. The net consequence was that adoption of methods was low during amenorrhea, particularly in Dhaka, and the onset of menses acted as a powerful trigger for initiation of use.

Studies coordinated by Family Health International in Ghana, India, Rwanda and Zambia give an impression about the importance of the return of menses as a signal to initiate contraceptive use similar to that of the SalwayNurani study. ${ }^{27}$ In three of these countries, between a quarter and a half of postpartum women were unaware that a woman could get pregnant before the return of menses. In Ghana and Zambia, among women who were amenorrheic and sexually active at 9-12 months postpartum, contraceptive use was no higher among women who were aware than it was among those who were unaware. In Rwanda, both survey and in-depth interviews revealed that amenorrhea was a major reason for non-use. ${ }^{28}$ Some women thought that they had to await the return of menses before they could request a contraceptive method and, indeed, some providers insisted on proof of menstruation.

However, belief in the protection represented by lack of menses does not seem universal in low- and middleincome populations. For low-income women in Istanbul in the early 1990s, menses was not a consideration in postpartum contraceptive adoption; only $2 \%$ reported that they awaited the return of menses before starting use. ${ }^{29}$ In Ouagadougou, Burkina Faso, semistructured interviews with 33 women suggested that belief in amenorrhea as a protection against pregnancy was nonexistent, though one-third considered the return of menses an important factor when planning contraceptive initiation, perhaps because of service-related restrictions on eligibility for contraceptive adoption. ${ }^{30}$

Emphatic generalizations from the behavioral evidence, together with the findings of qualitative enquiries, are unjustified. However six tentative conclusions are warranted. First, the postpartum phase is regarded as a time of vulnerability for mother and infant. Thus any perception that use of modern contraceptive methods may be a potential hazard to health acts as a powerful disincentive for early postpartum use. Second, reliance on the absence of menses as an indicator that conception is impossible or unlikely is widespread but not universal. Third, accurate information on risks of conception before the return of menses is low among mothers and probably among staff. Fourth, once menses have returned, breast-feeding is not a deterrent to contraceptive adoption, except in some countries where oral contraceptive use is common. Fifth, family planning 
staff may exacerbate confusion and delayed initiation of use by insisting on menstruation as evidence of nonpregnancy before offering methods. Finally, the current-status approach to assessing postpartum need for contraception is consistent with the behavior and beliefs of most women in low- and middle-income countries.

The relative merits of the current-status and prospective approaches to assessing postpartum need, and the programmatic implications of which approach is taken, depend to a large extent on local considerations. Among the 16 countries listed in Table 1 , the percentage of women at 6-11 months postpartum for whom menses or sex (or both) has not resumed ranges from $2 \%$ in Jordan to $64 \%$ in Ghana (results not shown). Obviously, a policy of regarding amenorrheic nonusers as needing contraception is much more appropriate in Jordan than in Ghana. Comparing levels of unmet need across populations in the first year postpartum is one obvious way of setting international priorities for investment in postpartum family planning provision. Under the prospective definition, the countries of Sub-Saharan Africa have the greatest need. Of the 16 countries in Table 1, the top six in terms of prospective unmet need are African. In contrast, the six countries with the highest current unmet need are Guyana, Pakistan, Uganda, the Dominican Republic, Kenya and Ghana.

Neither measure is satisfactory because no account is taken of fertility level. The number of births per woman is an important consideration in setting priorities because it defines the number of times that an average woman will require postpartum protection and the proportion of all children who run the risk of inadequate spacing with adjacent siblings. For instance, with a fertility rate of two, only half of children on average run the risk of being born within 24 months of an elder sibling. With a fertility rate of five, this proportion rises to $80 \%$. For this reason, it is preferable to establish priorities, not by unmet need measures, but by the straightforward expedient of multiplying the percentage of interbirth intervals that are shorter than 24 months by the total fertility rate (see Table 1). Under this approach to relative need, the top six countries are the DRC, Uganda, Pakistan, Nigeria, Jordan and Kenya. Guyana is now rated low in relative need because the high proportion of short intervals is offset by low fertility, while Ghana is rated low because high fertility is offset by a low proportion of short intervals.

\section{Differences in Unmet Need by Duration Postpartum Among Exposed Women}

As already established, unmet need is very low in the first year postpartum under the current-status definition, which classifies amenorrheic and abstaining women as having no need. However, the analysis thus far has not established the level of unmet need among postpartum women who have resumed sex and menstruation or how this level compares to the level among women at other stages of the reproductive cycle. Accordingly, we calculated unmet need for women who were fully exposed to the risk of concep-
FIGURE 2. Percentage of all exposed women who do not want a child within two years but are using no method, by duration postpartum, by region and country

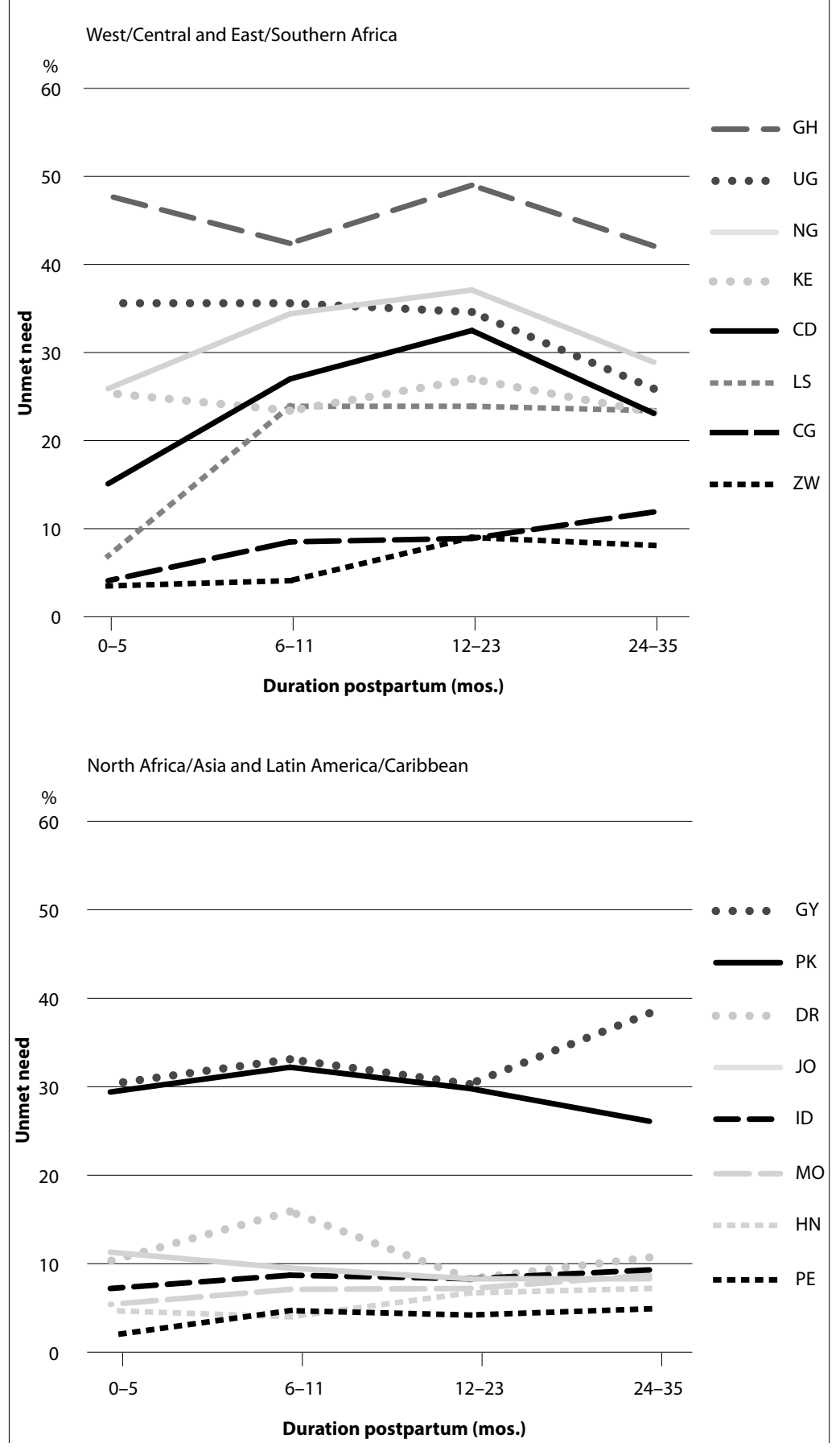

tion at the time of the survey by excluding nonusers who are still amenorrheic and have not resumed sex, as well as those who are pregnant. The results of this approach are shown in Figure 2. To be precise, this figure shows the percentage of women-among all married, fecund, nonpregnant women who have resumed menses and sex-who do not want a child within the next two years but are using no method of contraception. On a priori grounds, a steady decrease in unmet need is expected as duration postpartum (i.e., open interval length) increases because of selectivity. 
Women who remain without pregnancy or birth as duration lengthens are increasingly likely to be effective users of contraceptives.

Results for the eight surveys in Sub-Saharan Africa, shown in the upper panel, indicate a wide variety of patterns. In these countries, only a minority of women at 0-5 months postpartum have resumed menses and sex. However, among this minority, unmet need ranges from nearly 50\% in Ghana to about 5\% in Republic of Congo (Brazzaville) and Zimbabwe. These big differences largely reflect national variations in unmet need at all durations postpartum. In Nigeria and the DRC, unmet need rises steeply from the first six months to the second six months postpartum and continues to rise at 12-23 months before declining. In Lesotho, unmet need is also much higher at 6-11 months than earlier in the postpartum period, but levels off thereafter. In the Republic of Congo, Zimbabwe and Kenya, unmet need remains constant across all durations, at low levels in the first two countries and at a much higher level in the third. In Ghana, unmet need remains high at all durations, with no clear-cut trend while, in Uganda, it remains constant at about 35\% in the first two years postpartum, but declines to about $25 \%$ in the third year postpartum.

In view of this diversity, only one broad generalization can be made about trends in unmet need among exposed women by duration postpartum in these eight African countries. When attention is restricted to women fully exposed to risk of pregnancy, there is little support for the view that unmet need is particularly high in the first year postpartum. Indeed, the dominant pattern is that unmet need is higher in the second than in the first year postpartum and in none of these countries is it appreciably lower. The results for the eight surveys in other regions can be summarized in a single sentence. Unmet need does not vary by duration postpartum among exposed women, with a couple of minor exceptions in Guyana and the Dominican Republic

The analysis was redone with unmet need for a modern method as the outcome (results not shown). The general pattern was similar to that in Figure 2. It was also redone after broadening the definition of unmet need to include women who reported a current unintended pregnancy (results not shown). The predictable effect of this adjustment was to raise estimates of unmet need at longer durations postpartum, when pregnancy becomes more common. In seven of the eight countries of Sub-Saharan Africa, unmet need under this definition rose monotonically from 0-5 months to 12-23 months postpartum. In the other eight countries, trends were more similar than in Sub-Saharan Africa to those for fully exposed women.

This comparison of unmet need at different durations postpartum yields results that contrast strongly with those from the prospective approach of Ross and Winfrey, which found that 39\% of women who had given birth in the preceding 12 months have an unmet need. When the focus of attention is narrowed to women fully exposed to the risk of conception, unmet need tends to be lower in the first year postpartum than at longer durations. The important implication is that, once sex and menses have resumed, recently delivered mothers exhibit no greater reluctance to adopt methods of contraception than those at other stages of the reproductive cycle.

\section{Implications}

What sets family planning provision in the postpartum period apart from provision at other phases of the reproductive cycle is the degree of protection afforded by breastfeeding and, in a minority of countries, prolonged sexual abstinence. In Sub-Saharan Africa, most children are fed at the breast for 20 or so months and durations of postpartum insusceptibility from abstinence or amenorrhea, though shortening over time, typically remain close to 12 months. In other regions, durations of insusceptibility are much shorter, often only 3-4 months, but are more variable. In Asian countries covered by the DHS, the longest durations, of 8-9 months, are found in Cambodia, India, Nepal and Vietnam. In Latin America and the Caribbean, Bolivia, Ecuador, Guatemala, Haiti, Honduras and Peru record the longest durations (8-10 months).

The essential lesson to be drawn from this synthesis of the evidence is not to belittle the importance of postpartum family planning but to stress that strategies for family planning following childbirth need to take greater account of prevailing behavior and beliefs. In settings where lactational protection lasts on average for only 3-4 months, as is the case in six of the 16 countries considered in this paper, early uptake of contraceptives should be promoted without hesitation.

However, marked dilemmas about optimal postpartum strategies arise in Sub-Saharan Africa, and to a lesser extent in countries like India, where lactational amenorrhea typically extends through the first 12 months after childbirth. In such settings, three main options can be identified: to promote early uptake of LARC or sterilization; to counsel women about LAM and urge contraceptive adoption at six months or earlier; and to advise women accurately on the risks associated with exclusive reliance on lactational amenorrhea and inform them of other contraceptive options, while leaving choice on timing of contraceptive initiation to them. The first two options accord with established postpartum protocols. The third option, however, is a radical departure from these protocols.

The first option encounters the problem that use of LARC and permanent methods is rare in Africa and the popularization of unfamiliar methods is a major undertaking requiring training, logistics and demand creation. Research is needed to assess whether demand exists for immediate or early postpartum IUD, implant or sterilization services. Advocacy of early uptake of the most widely used methods, injectables and oral contraceptives, is illadvised for the majority of women because of high rates of discontinuation and low rates of switching to an alternative method. In African countries with relevant data, the 
median length of use is 10-11 months for the injectable and 10-16 months for the pill, except in Zimbabwe. Moreover, only minorities of African women switch to an alternative method after discontinuation. ${ }^{31}$ Adoption of these methods during, for instance, month five postpartum will have only a modest effect on postponement of the next pregnancy because of the high chance that the method will be discontinued before or shortly after resumption of ovulation.

The second option appears problematic because we see little prospect that counseling about LAM will be effective for most women, though the benefits for child health might be considerable. Exclusive breast-feeding, without liquid or solid supplements, is uncommon in most African countries after the first couple of months. ${ }^{32}$

Tabulations in DHS reports show that early supplementation with semisolid or solid foods is common and perhaps difficult to change. For instance, in Nigeria, Ghana, Uganda and Kenya, the percentage of children aged 4-5 months being fed such supplements ranges from 19\% to $38 \%$. Nevertheless, frequent breast-feeding remains the norm. More than $90 \%$ of mothers in most African DHS data sets report nursing children younger than six months of age at least six times a day. ${ }^{32}$ As discussed earlier, the chances of pregnancy among breast-feeding women in the first six months postpartum is very low, and diverting staff time to the complex task of explaining LAM with the aim of reducing pregnancy risks even further seems a poor investment of scarce staff time.

The third option is to explain to women the risks of relying on lactational amenorrhea and leave decisions to them. Such a post-amenorrheic strategy was once under serious consideration. ${ }^{33}$ Yet it is never mentioned as an option in more recent publications and recommendations. The precautionary principle of advocating highly effective methods of pregnancy prevention has obliterated consideration of the merits of somewhat less effective alternatives.

The evidence reviewed earlier suggests that sexually active, amenorrheic, breast-feeding women have a 3\% chance of pregnancy in the first six months after childbirth and a $6 \%$ chance in the first 12 months. Attitudes to this level of risk divide expert opinion. Kennedy and Visness concluded from their review that "it seems reasonable for a woman to rely on lactational amenorrhea without regard to whether she is fully or partly breast-feeding." 18 In contrast a Cochrane review of LAM encapsulates the prevailing consensus that "waiting for the end of amenorrhea before starting to use contraception is not acceptable." 34

Neither of these statements takes any account of the wishes of women or couples. In our view, a top research priority is to ascertain these wishes. At issue is the ability of women to fully grasp the probabilities of conception implied by a post-amenorrheic strategy and to assess their confidence that they will be able to obtain a method as soon as menses return. Qualitative research is appropriate for initial investigations. No doubt, the choices made will reflect the perceived consequences of pregnancy and perhaps access to medical or surgical abortion. One important principle of family planning services is to take into account the wishes and priorities of women and couples. We hope that this fresh look at postpartum contraception represents a small step toward this goal.

\section{REFERENCES}

1. Conde-Agudelo A, Rosas-Bermúdez A and Kafury-Goeta AC, Birth spacing and risk of adverse perinatal outcomes: a meta-analysis, Journal of the American Medical Association, 2006, 295(15):1809-1823.

2. United Nations Children's Fund (UNICEF) et al., Levels \& Trends in Child Mortality: Report 2013, New York: UNICEF, 2013.

3. Lawn JE et al., Every Newborn: progress, priorities, and potential beyond survival, Lancet, 2014, 384(9938):189-205.

4. Rutstein S, Further evidence of the effects of preceding intervals on neonatal, infant and under-five mortality and nutritional status in developing countries: evidence from the Demographic and Health Surveys, DHS Working Papers, Calverton, MD, USA: Macro International, 2008, No. 41.

5. Conde-Agudelo A, Rosas-Bermúdez A and Kafury-Goeta AC, Effects of birth spacing on maternal health: a systematic review, American Journal of Obstetrics \& Gynecology, 2007, 196(4):297-308.

6. Rutstein SO, Trends in birth spacing, DHS Comparative Reports, Calverton, MD, USA: ICF Macro, 2011, No. 28.

7. World Health Organization (WHO), Programming Strategies for Postpartum Family Planning, Geneva: WHO, 2013

8. Population Services International (PSI), Enabling the Healthy Spacing of Pregnancy: Programmatic Approaches to Expand Postpartum IUD Access, Washington, DC: PSI, 2015.

9. Ross JA and Winfrey WL, Contraceptive use, intention to use and unmet need during the extended postpartum period, International Family Planning Perspectives, 2001, 27(1):20-27.

10. Bradley $S$ et al., Revising unmet need for family planning, DHS Analytical Studies, Calverton, MD, USA: ICF International, 2012, No. 25.

11. Castadot $\mathrm{R}$ et al., The international postpartum family planning program: eight years of experience, Reports on Population/Family Planning, 1975, 18(1):1-53.

12. Borda M and Winfrey W, Postpartum Fertility and Contraception: An Analysis of Findings from 17 Countries, Baltimore, MD, USA: Jhpiego, 2010.

13. Bhatia S, Becker S and Kim YJ, The effect of oral contraceptive acceptance on fertility in the postpartum period, International Journal of Gynaecology \& Obstetrics, 1987, 25(Suppl.):1-11.

14. Becker S and Ahmed S, Dynamics of contraceptive use and breastfeeding during the post-partum period in Peru and Indonesia, Population Studies, 2001, 55(2):165-179.

15. Ngianga-Bakwin K and Stones RW, Birth intervals and injectable contraception in sub-Saharan Africa, Contraception, 2005, 71(5):353356 .

16. Short RV et al., Contraceptive effects of extended lactational amenorrhoea: beyond the Bellagio Consensus, Lancet, 1991, 337(8743):715-717.

17. Gray RH et al., Risk of ovulation during lactation, Lancet, 1990, 335(8680):25-29.

18. Kennedy KI and Visness CM, Contraceptive efficacy of lactational amenorrhoea, Lancet, 1992, 339(8787):227-230.

19. Thapa S, Kumar S and Cushing J, Contraceptive use and needs among postpartum women in 25 developing countries: recent patterns and implications, in: Proceedings of the Demographic and Health Surveys World Conference, 1991, Vol. 2, Columbia, MD, USA: Institute for Resource Development/Macro International, 1991, pp. 1149-1154.

20. Gebreselassie T, Rutstein S and Mishra V, Contraceptive use, breastfeeding, amenorrhea and abstinence during the postpartum period: an analysis of four countries, DHS Analytical Studies,

Calverton, MD, USA: Macro International, 2008, No. 14. 
21. Borda MR, Winfrey W and McKaig C, Return to sexual activity and modern family planning use in the extended postpartum period: an analysis of findings from seventeen countries, African Journal of Reproductive Health, 2010, 14(4 Spec no.):72-79.

22. Rai RK and Unisa S, Dynamics of contraceptive use in India: apprehension versus future intention among non-users and traditional method users, Sexual \& Reproductive Healthcare, 2013, 4(2):65-72.

23. Population Council, Increasing postpartum contraception in rural Uttar Pradesh: implications for behavior change communication, Policy Brief, New Delhi: Population Council, 2010, No. 7.

24. Haggerty P and Rutstein S, Breastfeeding and complementary infant feeding and the postpartum effects of breastfeeding, DHS Comparative Reports, Calverton, MD, USA: Macro International Inc, 1999, No. 30

25. Salway $S$ and Nurani S, Postpartum contraceptive use in Bangladesh: understanding users' perspectives, Studies in Family Planning, 1998, 29(1):41-57.

26. Salway S and Nurani S, Uptake of contraception during postpartum amenorrhoea: understandings and preferences of poor, urban women in Bangladesh, Social Science $\&$ Medicine, 1998, 47(7):899-909.

27. FHI 360, Postpartum Family Planning: New Research Findings and Program Implications, Research Triangle Park, NC, USA: FHI 360, 2012

28. Brunie A et al., Getting to 70\%: barriers to modern contraceptive use for women in Rwanda, International Journal of Gynaecology $\mathcal{E}$ Obstetrics, 2013, 123(Suppl. 1):el1-e15

29. Bulut A and Turan JM, Postpartum family planning and health needs of women of low income in Istanbul, Studies in Family Planning, 1995, 26(2):88-100

30. Rossier C and Hellen J, Traditional birthspacing practices and uptake of family planning during the postpartum period in Ouagadougou: qualitative results, International Perspectives on Sexual and Reproductive Health, 2014, 40(2):87-94.

31. Ali MM, Cleland J and Shah I, Causes and Consequences of Contraceptive Discontinuation: Evidence for 60 Demographic and Health Surveys, Geneva: WHO, 2012

32. Makuria A, Kothari M and Abderrahim N, Infant and Young Child Feeding Update, Calverton, MD, USA: ORC/Macro, 2006

33. Potter RG, Kobrin FE and Langsten RL, Evaluating acceptance strategies for timing of postpartum contraception, Studies in Family Planning, 1979, 10(5):151-160.

34. Van der Wijden C, Kleijnen J and Van den Berk T, Lactational amenorrhea for family planning, Cochrane Database of Systematic Reviews, 2003, Issue 4, No. CD001329.

Author contact:John.Cleland@lshtm.ac.uk 